\title{
microRNA-208a in an early stage myocardial infarction rat model and the effect on cAMP-PKA signaling pathway
}

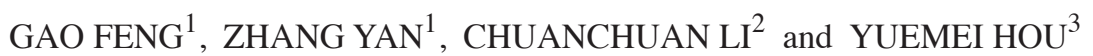 \\ ${ }^{1}$ The Third Clinical Medical College of Southern Medical University, Guangzhou, Guangdong 510515; \\ ${ }^{2}$ Fuzhou First Hospital Affiliated to Fujian Medical University, Fuzhou, Fujian 350000; \\ ${ }^{3}$ Fengxian Hospital Affiliated to Southern Medical University, Shanghai 201400, P.R. China
}

Received January 20, 2016; Accepted June 14, 2016

DOI: $10.3892 / \mathrm{mmr} .2016 .5402$

\begin{abstract}
The expression level of microRNA-208a (miR-208a) in a rat model with myocardial infarction and the effect of cAMP-PKA signaling pathway in early stage of myocardial infarction in rats were investigated. The early myocardial infarction model was established in 12 male Sprague-Dawley rats by ligation of the anterior descending coronary artery, and 12 rats were selected as the control group (sham operation group). Reverse-transcription quantitative PCR was conducted to detect the expression levels of miR-208a in the myocardium of and the expression levels of miR-208a in the serum of rats in the two groups. Western blot analysis was used to evaluate the expression levels of cAMP-PKA protein in the rat tissues in the two groups. After stimulating high levels of miR-208a expression in human myocardial cells (HCM), western blot analysis was used to detect the cAMP-PKA protein levels. The expression levels of miR-208a in myocardial tissues in rats with myocardial infarction were significantly higher than those in the control group, and the difference was statistically significant $(\mathrm{P}<0.05)$. The expression levels of miR-208a in the early stage of myocardial infarction rats were also significantly higher than those in the control group, and the difference was statistically significant $(\mathrm{P}<0.05)$. The level of cAMP-PKA protein in myocardial tissue in rats with chronic myocardial infarction was also significantly higher. Transfection of human myocardial cells with miR-208a analogue significantly increased the cAMP-PKA protein levels in human myocardial cells. In conclusion, the overexpression of miR-208a in myocardial infarction tissue and the high levels of this miRNA in the serum, may be involved in the process of myocardial
\end{abstract}

Correspondence to: Dr Gao Feng, The Third Clinical Medical College of Southern Medical University, Guangzhou, Guangdong 510515, P.R. China

E-mail: gmoh2167524@163.com

Key words: microRNA-208a, early myocardial infarction, cAMP, PKA infarction by influencing the cAMP-PKA signaling pathway in myocardial cells.

\section{Introduction}

MicroRNA (miRNA) is a small non-coding single-stranded RNA molecule with a length of approximately 22 nucleotides. miRNAs assume several key regulatory functions, such as cell growth, tissue differentiation, cell proliferation, embryonic development and cell apoptosis (1). The involvement of miRNAs in various tumors and cardiovascular diseases has been reported $(1,2)$. An abnormal miRNA level has been reported in many diseases including myocardial infarction (2). Results from those studies demonstrated that the abnormal expression was found uniquely in the case of specific miRNAs and their overexpression was used as genotype markers (3).

Early stage myocardial infarction is a serious health condition and timely diagnosis and treatment have an important impact on the quality of life of patients. Functional genomic research revealed that microRNA-208a (miR-208a) was solely expressed in the cardiac muscle cells, and released into the bloodstream after the occurrence of myocardial damage (4). However, to the best of our knowledge, no study reported the expression levels of miR-208a in myocardial infarction.

In this study, we examined the expression level of miR-208a in rat myocardial infarction tissue and the level of miR-208a in the serum. We also studied the possible effects of the miR-208a level on the cAMP-PKA pathway in myocardial injuries.

\section{Materials and methods}

Establishment of the early stage myocardial infarction rat model. Male Sprague-Dawley rats weighing 200 to $220 \mathrm{~g}$, were obtained from the Experimental Animal Center of Fengxian Hospital (Shanghai, China), were divided into two groups. Rats in the sham operation group were given thoracotomy without ligation of coronary artery, while rats in the myocardial infarction group were fed for another 8 weeks after ligation of anterior descending coronary artery. After administration of chloral hydrate $(10 \%, 350 \mathrm{mg} / \mathrm{kg})$ to rats in 
the myocardial infarction group under anesthesia, the neck skin was incised longitudinally, while limbs and head were fixed in the supine position, exposing the trachea after blunt separation. The trachea was cut between the second and third cricoid cartilage and the animal ventilator was inserted into the trachea after intubation for artificial respiration. Skin was incised in the 3rd to 4th intercostal along the rib gap on the left side of the sternum. Subcutaneous tissue and muscle were then separated layer by layer. The chest was entered in the 3rd to 4th intercostal to expose the heart. Initial portion of the left anterior descending coronary artery was located between the left atrial appendage margin and the pulmonary cone. The left anterior descending coronary artery was ligated with a wisp of myocardia. After ligation, the left ventricular wall turned pale, and ventricular wall motion decreased.

The rat model. The ST segment of lead II in ECG monitor was significantly higher. Chest was monitored for $10 \mathrm{~min}$ and then closed layer by layer after thorough hemostasis. Spontaneous breathing was recovered and the trachea as well as the neck incisions were sutured. Rats in the sham operation group were only treated with open thoracic puncture needle without ligation of the coronary artery. After the experiment, rats in the sham operation group were in good condition with smooth hair and were active and agile. The nutritional status of rats in the myocardial infarction group was poor. The hair was messy and sparse, and the movement of the rats was slow with less activity.

Ethics approval for the animal experiments was received from the Medical Ethics Committee of Fengxian Hospital.

\section{Experimental method}

Total RNA extraction, serum collecting and reverse transcription reactions

Serum extraction. Peripheral venous blood was collected from the two groups and EDTA anticoagulant was added to the samples. Samples were centrifuged at 4,000 x $\mathrm{g}$ for $5 \mathrm{~min}$ at $4^{\circ} \mathrm{C}$. After centrifugation, the serum was kept at $-80^{\circ} \mathrm{C}$. TRIzol (1 ml; Gibco, Carlsbad, CA, USA) was added to approximately $100 \mathrm{mg}$ of tissue or $100 \mu \mathrm{l}$ of serum. Samples were homogenized and total RNA was extracted. Extracted RNA was dissolved in $30 \mu 1 \mathrm{DEPC}$ water and reverse transcription kit (Thermo Fisher Scientific, Waltham, MA, USA) was used for reverse transcription.

PCR reaction conditions. PCR was set for 40 cycles (denaturation at $95^{\circ} \mathrm{C}$ for $20 \mathrm{sec}$, renaturation at $60^{\circ} \mathrm{C}$ for $20 \mathrm{sec}$ and extension at $70^{\circ} \mathrm{C}$ for $1 \mathrm{sec}$ ). Primers used were: $m i R-208 a$ forward, 5'-GTCATCTAGAAAGCTTGATGCAGGAAA GAGCTTTGG-3' and reverse, 5'-TGACAGATCTCAGCTGA CATCCTCTAGGCTGGGGTT-3' (5); U6 control forward, 5'-CTCGCTTCGGCAGCACA-3' and reverse 5'-AACGCT TCACGAATTTGCGT-3'.

PCR was conducted with ABI PCR, model 7900 (Applied Biosystems, Foster City, CA, USA). The relative quantitative analysis was performed using the $2^{-\Delta \Delta C t}$ method (6).

Cell transfection. Lipofectamine 2000 (Invitrogen, Carlsbad, CA, USA) was used for myocardial cell line transfection using miR-208a stimulant (mimic; Guangzhou Ruibo Biotechnology Co., Ltd., Guangzhou, China). The interference efficiency was evaluated after $48 \mathrm{~h}$.
Table I. Expression levels of miR-208a in rat myocardial tissues.

\begin{tabular}{lcc}
\hline Group (n) & miR-208a miRNA & t/P-value \\
\hline Control (12) & $1.21 \pm 0.31$ & \\
Study (12) & $4.21 \pm 1.41$ & $7.20 / 0.000^{a}$ \\
\hline
\end{tabular}

${ }^{\mathrm{a} C o m p a r e d}$ with the rat myocardial tissue in the control group. $\mathrm{t}=7.20$, $\mathrm{P}<0.05$. miR-208a, microRNA-208a; miRNA, microRNA.

Table II. miR-208a levels in the serum.

\begin{tabular}{lcc}
\hline Group (n) & miR-208a miRNA & t/P-value \\
\hline Control (12) & $1.29 \pm 0.41$ & \\
Study (12) & $3.24 \pm 1.39^{\mathrm{a}}$ & $4.66 / 0.000$ \\
\hline
\end{tabular}

${ }^{\mathrm{a} C o m p a r e d}$ with the rat myocardial tissue in the control group. $\mathrm{t}=7.20$, $\mathrm{P}<0.05$. miR-208a, microRNA-208a; miRNA, microRNA.

Evaluation of protein expression by western blotting. HCM cells were collected and treated with 1X SDS cell lysis buffer (Beyotime Inc., Shanghai, China). After SDS-PAGE, the proteins were transferred to the PVDF membrane (Millipore, Shanghai, China) at $110 \mathrm{~V}$ for $90 \mathrm{~min}$. The membranes were blocked at $37^{\circ} \mathrm{C}$ for $100 \mathrm{~min}$ and then incubated with polyclonal goat anti-human PKA (R\&D Systems, Inc., Minneapolis, MN, USA cat no. AF4177, 1:500 dilution) and monoclonal mouse anti-human cAMP antibody (R\&D Systems, Inc., Minneapolis, MN, USA , cat no. MAB2146, 1:500 dilution) at $4^{\circ} \mathrm{C}$ overnight. Subsequently, the membranes were rinsed and incubated with anti-goat secondary (PKA, cat. no. ab339770) and HRP-mouse secondary (cAMP, cat. no. ab157532) antibodies (1:2,000; Abcam, Cambridge, MA, USA) at $37^{\circ} \mathrm{C}$ for $30 \mathrm{~min}$. The reference protein used in this study was rabbit polyclonal to human $\beta$-actin (ab8227, 1:500) (Sigma, St. Louis, MO, USA).

Statistical analysis. Data were analyzed using SPSS 19.0 software (SPSS software Inc., Chicago, IL, USA). The differences between the two groups were compared using the Student's t-test. $\mathrm{P}<0.05$ was considered statistically significant.

\section{Results}

Expression levels of miR-208a in the rat myocardial tissues. The expression levels of miR-208a in myocardial tissue in rats with early myocardial infarction were significantly higher than those in control group $(\mathrm{P}<0.05)$ (Table I).

miR-208a levels in the serum. For rats with subacute myocardial infarction we detected higher levels of miR-208a in the serum. The difference observed between the myocardial infarction and the control group was statistically significant $(\mathrm{P}<0.05)$ (Table II).

cAMP-PKA expression levels in the rat myocardial tissues. The expression levels of cAMP-PKA protein in the myocardial 
A
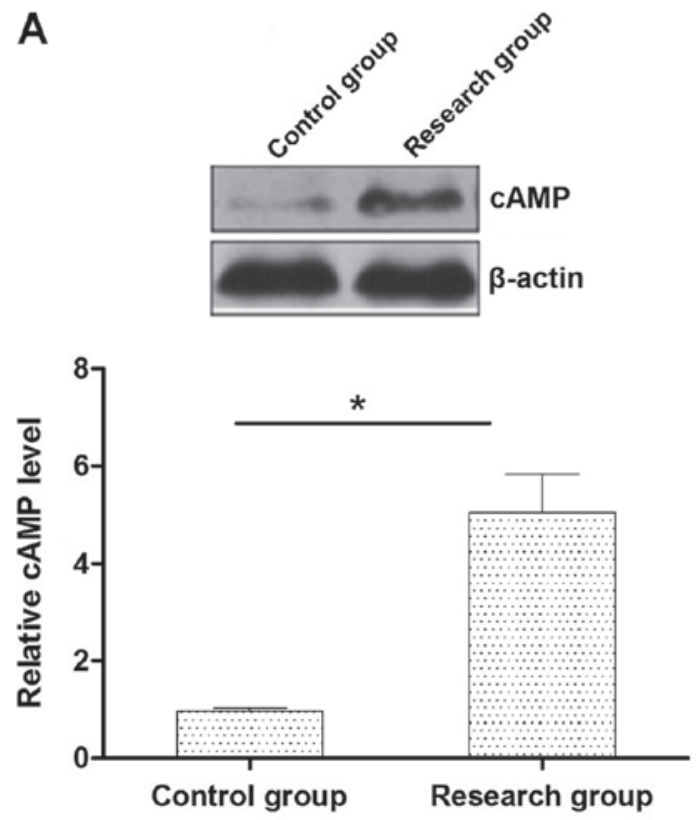

B
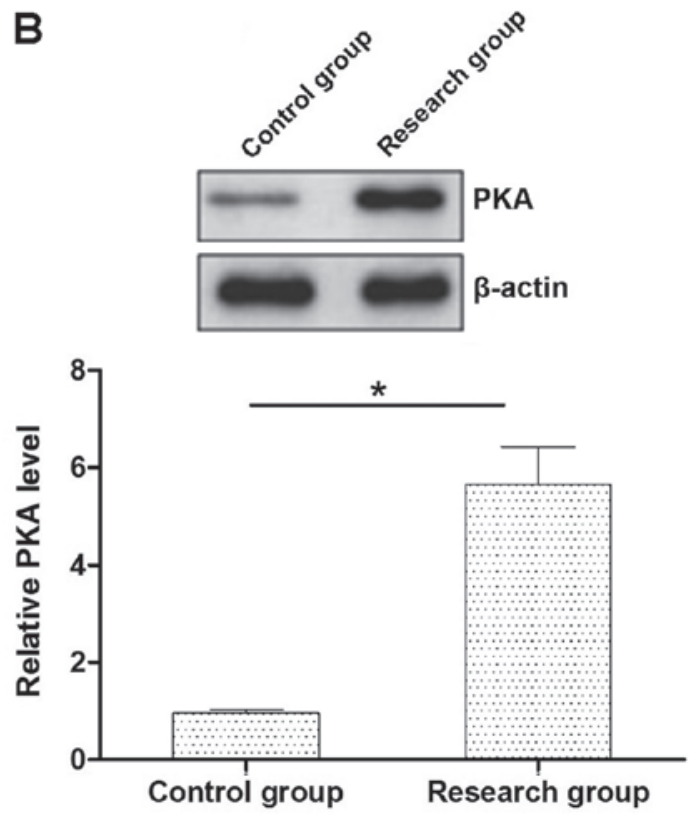

Figure 1. The cAMP-PKA protein expression levels in the rat myocardial tissues (both groups). ${ }^{*} \mathrm{P}<0.05$, compared with the control group.

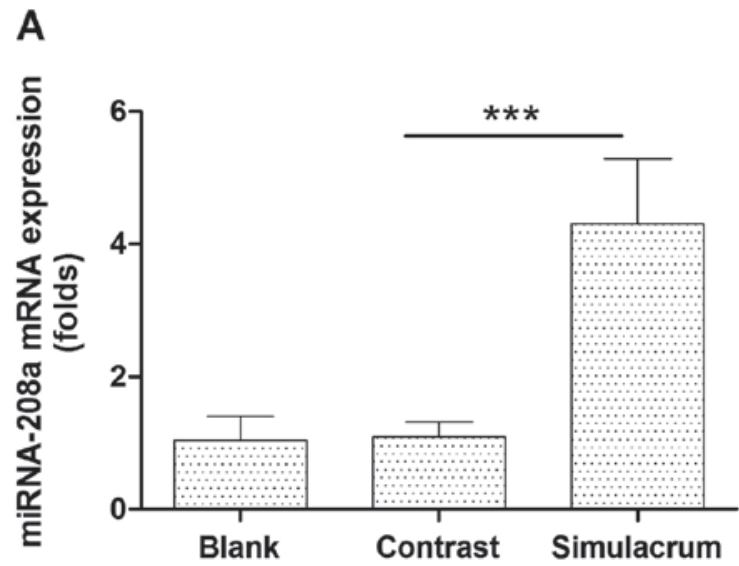

B

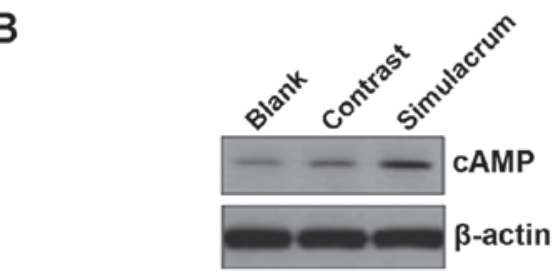

C
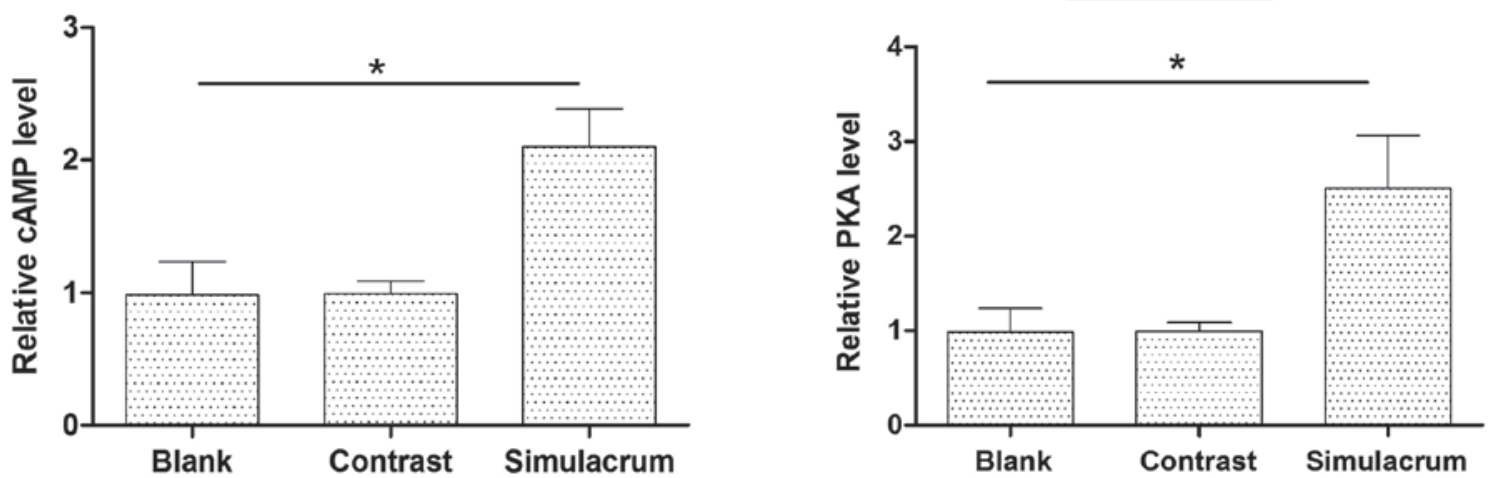

Figure 2. The effect of miR-208a stimulation on the expression levels of the miR-208a (A). The effect of miR-208a overexpression on cAMP-PKA levels in human myocardial cells (B and C). ${ }^{*} \mathrm{P}<0.05$, compared with the blank group; ${ }^{* * *} \mathrm{P}<0.005$, compared with the blank group. miR-208a, microRNA-208a. 
tissue in rats with early myocardial infarction were significantly higher than those in the control group $(\mathrm{P}<0.05)$ (Fig. 1).

The effects of miR-208a overexpression on the cAMP-PKA expression levels in cardiac muscle cells. Transfecting cells with the miR-208a stimulation increased the expression levels of the miR-208a (Fig. 2A). High levels of miR-208a expression significantly upregulated the cAMP-PKA protein expression (Fig. 2B and C).

The differences observed between the experimental and control groups were statistically significant.

\section{Discussion}

Acute myocardial infarction is a multi-factor related disease on the basis of atherosclerosis. Its clinical manifestation is intense and persistent chest pain. Resting or taking nitrates usually cannot induce complete remission. Acute myocardial infarction is often accompanied by an increase in serum myocardial enzyme and troponin activity. We also observed severe and dangerous electrocardiogram changes. Acute myocardial infarction can cause severe damage to patients and has a high disability rate as well as high mortality rate (7). Effective and timely medical intervention is an important element to reduce the devastating impact of myocardial infarction and to improve the survival rates of the patients suffering from this condition. At present, laboratory diagnosis of acute myocardial infarction is obtained by measuring serum myocardial damage markers (myocardial enzymes and troponin) (2). However, there are some shortcomings, such as poor specificity and short duration. Therefore, it is imperative to identify a more specific marker for the treatment and prognosis of acute myocardial infarction.

miRNA is a class of single-stranded short chain nonencoding small RNA fragments with regulatory effects on human genes. miRNAs is important in the process of cell differentiation and apoptosis (8-12). In recent years, the importance of miRNA as an endogenous factor in myocardial ischemia has been demonstrated. Prior studies showed that miRNA was involved in the pathological process of myocardial infarction and fibrosis process after myocardial infarction. For example, it was found that miR-199a levels in a rat model of acute myocardial infarction tissue decreased significantly and were involved in the regulation of myocardial hypoxia by regulating the hypoxia-inducible factor (HIF-1 $\alpha$ ) expression level $(13,14)$. Rane et al found that miR-21, miR-214 and miR-223 in the myocardial infarction margin in patients with myocardial infarction increased significantly, while miR-29b and miR-149 significantly decreased (14). Previous genomic studies demonstrated that miR-208a exists in the human body, and its expression in patient peripheral blood following myocardial infarction was increased (4). To the best of our knowledge, there are only few studies that focus on the level of miR-208a in animal models for acute myocardial infarction and the mechanism of myocardial injury.

In the present study, the expression levels of miR-208a in the serum as well as the tissue were detected in an acute myocardial infarction rat model. The aim was to examine the function and mechanism of miR-208a involvement in the pathogenesis of acute myocardial infarction. The levels of miR-208a in the myocardial tissue and the serum were significantly higher in rats with acute myocardial infarction suggesting that miR-208a may be involved in the occurrence and development of acute myocardial infarction. We hypothesized that miR-208a is released into the blood after myocardial infarction. This is consistent with a previous study conducted on patients with myocardial infarction (4).

The cAMP-PKA pathway is a part of the receptor $\mathrm{G}$ protein and cAMP-PKA signaling pathway, which plays a key role in mediating cell responses to various stimuli (15). The cAMP-PKA pathway can mediate a variety of physiological and pathological functions, especially in cardiovascular disease, such as the expansion of coronary artery and enhanced myocardial contractility (16). The results of this study showed that cAMP-PKA protein levels in myocardial tissue were significantly higher in rats with subacute myocardial infarction compared to those in sham-operated rats. This findings showed that myocardial injury was involved in the occurrence of myocarditis by promoting the cAMP-PKA protein signaling pathway. Furthermore, the effect of miR-208a on the cAMP-PKA protein signaling pathway in myocardial cells was studied by overexpressing the miR-208a in human myocardial cells. It was found that the overexpression of miR-208a, significantly upregulated the cAMP-PKA expression. This result suggests that miR-208a is probably involved in the inflammatory process of acute myocardial infarction by affecting the cAMP-PKA signaling pathway.

In conclusion, we have demonstrated that the expression of miR-208a increased during the acute myocardial infarction and may be involved in the development of myocardial infarction by regulating the cAMP-PKA signaling pathway. We also suggest that our study showed that the detection of miR-208a in the serum of patients with subacute myocardial infarction can be used as a potential diagnostic indicator/marker for myocardial injuries.

\section{References}

1. Li Z, Ying X, Chen H, Ye P, Shen Y, Pan W and Zhang L: MicroRNA-194 inhibits the epithelial-mesenchymal transition in gastric cancer cells by targeting FoxM1. Dig Dis Sci 59: 2145-2152, 2014.

2. Lewis BP, Burge CB and Bartel DP: Conserved seed pairing, often flanked by adenosines, indicates that thousands of human genes are microRNA targets. Cell 120: 15-20, 2005.

3. Cheng Y, Liu X, Yang J, Lin Y, Xu DZ, Lu Q, Deitch EA, Huo Y, Delphin ES and Zhang C: MicroRNA-145, a novel smooth muscle cell phenotypic marker and modulator, controls vascular neointimal lesion formation. Circ Res 105: 158-166, 2009.

4. Suárez Y, Fernández-Hernando C, Pober JS and Sessa WC: Dicer dependent microRNAs regulate gene expression and functions in human endothelial cells. Circ Res 100: 1164-1173, 2007.

5. Callis TE, Pandya K, Seok HY, Tang RH, Tatsuguchi M, Huang ZP, Chen JF, Deng Z, Gunn B, Shumate J, et al: MicroRNA-208a is a regulator of cardiac hypertrophy and conduction in mice. J Clin Invest 119: 2772-2786, 2009.

6. Livak KJ and Schmittgen TD: Analysis of relative gene expression data using real-time quantitative PCR and the 2(-Delta Delta C(T)) method. Methods 25: 402-408, 2001.

7. Newby AC: Metalloproteinases promote plaque rupture and myocardial infarction: A persuasive concept waiting for clinical translation. Matrix Biol 44-46:157-66, 2015.

8. Zhang Y, Zhou ZG, Wang L, Zhang P, Wang MJ, Cui CF, Guan JT, Chen KL and Zhan L: Clinicopathological significance of microRNA-21 and miR-125 expression in colorectal cancer. Zhonghua Wei Chang Wai Ke Za Zhi 12: 623-626, 2009 (In Chinese). 
9. Du J, Yang S, An D, Hu F, Yuan W, Zhai C and Zhu T: BMP-6 inhibits microRNA-21 expression in breast cancer through repressing deltaEF1 and AP-1. Cell Res 19: 487-496, 2009.

10. Zhang JG, Wang JJ, Zhao F, Liu Q, Jiang K and Yang GH MicroRNA-21 (miR-21) represses tumor suppressor PTEN and promotes growth and invasion in non-small cell lung cancer (NSCLC). Clin Chim Acta 411:846-852, 2010.

11. Bae HJ, Noh JH, Kim JK, Eun JW, Jung KH, Kim MG, Chang YG Shen Q, Kim SJ, Park WS, et al: MicroRNA-29c functions as a tumor suppressor by direct targeting oncogenic SIRT1 in hepatocellular carcinoma. Oncogene 33: 2557-2567, 2014.

12. Fan DN, Tsang FH, Tam AH, Au SL, Wong CC, Wei L, Lee JM, $\mathrm{He} \mathrm{X}, \mathrm{Ng}$ IO and Wong CM: Histone lysine methyltransferase, suppressor of variegation 3-9 homolog 1, promotes hepatocellular carcinoma progression and is negatively regulated by microRNA-125b. Hepatology 57: 637-647, 2013.
13. Zhu H and Fan GC: Role of microRNAs in the reperfused myocardium towards post-infarct remodelling. Cardiovasc Res 94: 284-292, 2012.

14. Rane S, He M, Sayed D, Vashistha H, Malhotra A, Sadoshima J, Vatner DE, Vatner SF and Abdellatif M: Downregulation of miR-199a derepresses hypoxia-inducible factor-1alpha and Sirtuin 1 and recapitulates hypoxia preconditioning in cardiac myocytes. Circ Res 104: 879-886, 2009.

15. Raghunandan R and Ingram VM: Hyperphosphorylation of the cytoskeletal protein Tau by the MAP-kinase PK40erk2: regulation by prior phosphorylation with cAMP-dependent protein kinase A. Biochem Biophys Res Commun 215: 1056-1066, 1995.

16. Fantidis P: The role of intracellular 3'5'-cyclic adenosine monophosphate (cAMP) in atherosclerosis. Curr Vasc Pharmacol 8: 464-472, 2010 . 